\title{
Fluorescence-based immunoassay for the detection of Xanthomonas oryzae pv. oryzae in rice leaf
}

\begin{abstract}
The identification of rice bacterial leaf blight disease requires a simple, rapid, highly sensitive, and quantitative approach that can be applied as an early detection monitoring tool in rice health. This paper highlights the development of a turn-off fluorescence-based immunoassay for the early detection of Xanthomonas oryzae pv. oryzae (Xoo), a gram-negative bacterium that causes rice bacterial leaf blight disease. Antibodies against Xoo bacterial cells were produced as specific biorecognition molecules and the conjugation of these antibodies with graphene quantum dots and gold nanoparticles was performed and characterized, respectively. The combination of both these bio-probes as a fluorescent donor and metal quencher led to changes in the fluorescence signal. The immunoreaction between AntiXoo-GQDs, Xoo cells, and AntiXoo-AuNPs in the immunoaggregation complex led to the energy transfer in the turn-off fluorescence-based quenching system. The change in fluorescence intensity was proportional to the logarithm of Xoo cells in the range of 100-105 CFU mL-1. The limit of detection was achieved at $22 \mathrm{CFU} \mathrm{mL}-1$ and the specificity test against other plant disease pathogens showed high specificity towards Xoo. The detection of Xoo in real plant samples was also performed in this study and demonstrated satisfactory results.
\end{abstract}

Keyword: Fluorescence switch; Antibody; Graphene quantum dots; Gold nanoparticles; Rice bacterial leaf blight disease 